\title{
An Efficient Algorithm and Its Parallelization for Computing PageRank
}

\author{
Jonathan Qiao, Brittany Jones, and Stacy Thrall \\ Converse College \\ Spartanburg, SC 29302, USA \\ \{Jonathan.Qiao, Brittany. Jones, Stacy. Thrall\}@converse.edu
}

\begin{abstract}
In this paper, an efficient algorithm and its parallelization to compute PageRank are proposed. There are existing algorithms to perform such tasks. However, some algorithms exclude dangling nodes which are an important part and carry important information of the web graph. In this work, we consider dangling nodes as regular web pages without changing the web graph structure and therefore fully preserve the information carried by them. This differs from some other algorithms which include dangling nodes but treat them differently from regular pages for the purpose of efficiency. We then give an efficient algorithm with negligible overhead associated with dangling node treatment. Moreover, the treatment poses little difficulty in the parallelization of the algorithm.
\end{abstract}

Keywords: PageRank, power method, dangling nodes, algorithm.

\section{Introduction}

A significant amount of research effort has been devoted to hyperlink analysis of the web structure since Sergey Brin, Larry Page brought their innovative work [1] to the information science community in 1998. Brin and Page launched Google at the time when there were already a number of search engines. Google has succeeded mainly because it has a better way of ranking web pages, which is called PageRank by its founders.

The original PageRank model is solely based on the hyperlink structure. It considers the hyperlink structure of the web as a digraph. For each dangling node (a page without any out-link), edges are added so that it is connected to all nodes. Based on this, an adjacency matrix can be obtained. It is then to find the eigenvector of the adjacency matrix, whose elements are the ranking values of the corresponding web pages. Due to the size of the web graph, the dominant way in finding the eigenvector is the classic power method 2, which is known for its slow convergence. Because of this, a large amount of work has been done to speed up the PageRank computation since 1998.

One factor contributing substantially to the computational cost is the existence of dangling nodes. A single dangling node adds a row full of ones to the adjacency matrix. Including dangling nodes not only produces a significantly 
larger matrix, but also makes it no longer sparse. Some existing algorithms exclude dangling nodes from consideration in order to speed up the computation. Those which consider them don't treat them as regular pages for purpose of efficiency. In either case, the original web structure is changed, and more importantly the information carried by dangling nodes is changed as well.

In this paper, we will aim at finding the PageRank vector using the power method with dangling nodes fully included and treated as regular pages. We will then propose an highly efficient algorithm which minimizes the overhead of our treatment of dangling nodes to be negligible in either centralized or distributed approach.

\section{PageRank Review}

As discussed in Sect. 11 the web structure is treated as a digraph, which gives a $N \times N$ column-wise adjacency matrix, where $N$ is the number of total web pages. Let the matrix be $A$. Let $D$ be a $N \times N$ diagonal matrix with each entry the reciprocal of the sum of the corresponding column of $A$. Then, $A D$ is a column stochastic Markov matrix. Let $\mathbf{v}=A D \mathbf{e}$, where $\mathbf{e}$ is a vector of all ones. The $i$ th entry of $\mathbf{v}$ tells how many times the $i$ th page gets recommended by all web pages. Introducing the weight of a recommendation made by a page which is inversely proportional to the total number of recommendations made by that page gives the equation

$$
\mathbf{v}=A D \mathbf{v}
$$

Equation (11) suggests that $\mathbf{v}$ is the ranking vector as well as an eigenvector of $A D$ with the eigenvalue 1 .

By nature of the web structure, the Markov matrix $A D$ is not irreducible [2]. This implies that there are more than one independent eigenvectors associated with the eigenvalue 1 . Google's solution to this problem is to replace $A D$ by a convex combination of $A D$ and another stochastic matrix $\mathbf{e e}^{T} / N$,

$$
\mathbf{v}=\left(\alpha A D+\frac{1-\alpha}{N} \mathbf{e e}^{T}\right) \mathbf{v}
$$

where $0<\alpha<1$ (Google uses $\alpha=0.85$ ). The underlying rational is, by probability 0.85 , a random surfer chooses any out-link on a page arbitrarily if the page has out-links; by probability 0.15 , or if a page is a dead end, another page is chosen at random from the entire web uniformly.

The convex combination in (2) makes the ranking vector $\mathbf{v}$ the unique eigenvector associated with the eigenvalue 1 up to scaling and the subdominant eigenvalue $\alpha$, which is the rate of convergence when the power method is applied 3,42 . By normalizing the initial and subsequent vectors, a new power method formulation of (2) is

$$
\mathbf{v}^{i+1}=\alpha A D \mathbf{v}^{i}+\frac{1-\alpha}{N} \mathbf{e}
$$

where $\mathbf{v}^{0}=(1 / N)$ e because $\mathbf{e}^{T} \mathbf{v}=1$. 


\section{Related Work}

Most algorithms so far focus on the cost reduction of each power method iteration, although there have been efforts aiming at reducing the number of iterations, such as 5. Because the major obstacle of speeding up each iteration lies in the size of data, it is natural to compress the data such that it fits into the main memory, as by 678]. However, as pointed out by [6, even the best compression scheme requires about .6 bytes per hyperlink, which still results in an exceedingly large space requirement. Others are to design I/O-efficient algorithms, such as 910, which can handle any size of data without any particular memory requirement. Also aiming at reducing the cost of each iteration, many other works, such as 1112, combine some linear algebra techniques to reduce the computational cost of each iteration. Nevertheless, the I/O-efficiency must always be considered.

In [9], Haveliwala proposes two algorithms, Naive Algorithm and Blocking Algorithm, and demonstrates high I/O efficiency of the latter when the ranking vector does not fit in the main memory. Applying the computational model in (3), both algorithms use a binary link file and two vectors, the source vector holding the ranking values for the iteration $i$ and the destination vector holding the ranking values for the iteration $i+1$. Each diagonal entry of the matrix $D$ is stored in the corresponding row of the link file as the number of out-links of each source page.

For purpose of the cost analysis, there are several more parameter families, $M, B(\cdot), K$ and $n n z(\cdot)$.

1. The total number of available memory pages will be denoted $M$.

2. The total number of pages of disk/memory resident will be denoted $B(\cdot)$.

3. The total number of dangling nodes will be denoted $K$.

4. The total number of links of a web digraph will be denoted $n n z(\cdot)$.

Unless specified, the cost is for a single iteration since we focus on reducing the cost of each iteration in this work.

\subsection{Matrix-Vector Multiplication Treatment}

Given the computation model in (3), computing a single entry of $\mathbf{v}^{i+1}$ requires reading a row of matrix $A$ and the source vector. Since the link file is sorted by source node $i d$ 's and all elements of a row of matrix $A$ are spread out in the link file1, a less careful implementation would result in one pass for calculating a single value. Preprocessing the link file so that it is sorted by destination node $i d$ 's not only needs tremendous effort, but also adds $N$ entries for each dangling node to the link file and thus significantly increases the storage requirement and the I/O cost. To get around of this difficulty, we could use the column version of the matrix-vector multiplication

$$
A \mathbf{v}=v_{0} A_{* 0}+v_{1} A_{* 1}+\cdots+v_{n-1} A_{*(n-1)},
$$

${ }^{1}$ A column of $A$ corresponds to a row in the adjacency list. 
where each $A_{* i}$ is the $i$ th column of $A$. The computation model in (4) requires one pass of the link file and the source vector for finding the destination vector when the destination vector can be a memory resident.

\subsection{The Blocking Algorithm}

Let $\mathbf{v}^{\prime}$ be the destination vector, $\mathbf{v}$ be the source vector and $L$ be the adjacency list of the link file. To handle the memory bottleneck, Blocking Algorithm in 9 partitions $\mathbf{v}^{\prime}$ evenly into $b$ blocks so that each $\mathbf{v}_{i}^{\prime}$ fits in $(M-2)$ pages. $L$ is vertically partitioned into $b$ blocks. Note, each $L_{i}$ is then represented by a horizontal block $A_{i}$. The partition gives the equation

$$
\mathbf{v}_{i}^{\prime}=\alpha A_{i} D \mathbf{v}+\frac{1-\alpha}{N} \mathbf{e},
$$

where $i \in\{0,1, \cdots, b-1\}$.

Based on (5), computing each $\mathbf{v}_{i}^{\prime}$ requires one pass of $\mathbf{v}_{i}^{\prime} L_{i}, \mathbf{v}$. Updating the source vector at the end adds another pass of $\mathbf{v}$. Therefore, the cost (referred to as $\left.C_{\text {block }}\right)$ is

$$
C_{\text {block }}=\sum_{i=0}^{b-1} B\left(L_{i}\right)+(b+1) B(\mathbf{v})+\sum_{i=0}^{b-1} B\left(\mathbf{v}_{i}^{\prime}\right)=(b+2) B(\mathbf{v})+(1+\epsilon) B(L)
$$

where $\epsilon$ is a small positive number due to the partition overhead.

The cost in (6) does not scale up linearly when $b$ is not a constant, which can happen given the remarkably fast growth of the web repository.

\section{Dangling Nodes Treatment}

The cost model in (6) only counts disk I/O's with an assumption that the inmemory computational cost is negligible compared to the I/O cost. The assumption is justifiable when $A$ is sparse since the in-memory cost is approximately the number of 1 's in $A$. This can be seen in 9], whose test data set which contains close to $80 \%$ of dangling nodes originally is preprocessed to exclude all dangling nodes.

Many web pages are by nature dangling nodes, such as, a PDF document, an image, a page of data, etc. In fact, dangling nodes are a increasingly large portion of the web repositories. For some subsets of the web, they are about $80 \%$ [2]. Some dangling nodes are highly recommended by many other important pages, simply throwing them away may result in a significant loss of information. This is why some existing works don't exclude dangling nodes, such as [12, which computes the ranking vector in two stages. In the first stage, dangling nodes are lumped into one; in the second stage, non-dangling nodes are combined into one. The global ranking vector is formed by concatenating two vectors.

One of goals of this work is to make improvements over [9] with dangling nodes included. Different from 12 and some other algorithms which include dangling nodes, our approach treats them as regular web pages. It can be easily 
seen that including dangling nodes does not add any storage overhead since a dangling node does not appear in the link file as a source $i d$. Thus, our approach does not change the $\mathrm{I} / \mathrm{O}$ cost model in (6).

To minimize the in-memory computational overhead imposed by inclusion of dangling nodes, we decompose $A$ into $\hat{A}+\mathbf{e} \Delta^{T}$, where $\hat{A}$ is an adjacency matrix of the original web graph (a row full of zeros for a dangling node), $\Delta$ is a $N \times 1$ vector with the $i$ th entry 1 if the $i$ th node is a dangling node and 0 otherwise. Substituting the decomposition of $A$ into (3), we have

$$
\mathbf{v}^{i+1}=\alpha\left(\hat{A}+\mathbf{e} \Delta^{T}\right) D \mathbf{v}^{i}+\mathbf{c}=\alpha \hat{A} D \mathbf{v}^{i}+\mathbf{c}^{i}+\mathbf{c},
$$

where $\mathbf{c}=\mathbf{e}(1-\alpha) / N$, a constant vector at all iterations, $\mathbf{c}^{i}$ is a constant vector at iteration $i$, whose constant entry is computed by adding all dangling node ranking values at the beginning of each iteration, which is

$$
\sum_{\text {out }- \text { degree }(i)=0}(D \mathbf{v})_{i} .
$$

This involves $K$ (the number of dangling nodes) additions and one multiplication. In the implementation, we extract out-degree's from every $L_{i}$ and save them in a separate file, which has $N$ entries and can be used for computing (8). This also has an advantage of reducing the storage overhead caused by partition since each nonzero out-degree repeatedly appears in every $L_{i}$.

A substantial gain of the in-memory cost can be achieved. Let $\mathcal{G}$ be an original web digraph, then, the total number of floating number additions in (3) is

$$
C_{1}=n n z(\mathcal{G})+K N+N=(r+K+1) N,
$$

where $r$ is the average out-links, which varies from 5 to 15 [10]. Based on (77), this cost can be reduced to

$$
C_{2}=n n z(\mathcal{G})+K=r N+K .
$$

When $K$ is large compared to $N$, which is often the case for the web data, $C_{1}$ is $\Theta\left(N^{2}\right)$ while $C_{2}$ is $\Theta(N)$.

\section{The Parallelization of the Algorithm}

The computation model in (77) can be readily parallelized without any extra parallel overhead associated with inclusion of dangling nodes.

When applying Blocking Algorithm directly, we may vertically partition the link file $L$ into $L_{0}, L_{1}, \cdots, L_{b-1}$ and distribute them over $b$ nodes in a cluster. Each node holds a partition of $L$, the source vector $\mathbf{v}$ and a partition of $\mathbf{v}^{\prime}$. This gives the computation model at each node

$$
\mathbf{v}_{i}^{\prime}=\alpha A_{i} D \mathbf{v}+\mathbf{c}_{i}
$$

where $\mathbf{c}_{\mathbf{i}}$ is a constant vector of the size $N / b$ with $(1-\alpha) / N$ for all entries. 
The above parallelization does not take the advantage given by the dangling nodes treatment. Combining (5) and (7), the new parallel formulation can be established as

$$
\mathbf{v}_{i}^{\prime}=\alpha A_{i} D \mathbf{v}+\hat{\mathbf{c}}_{i}+\mathbf{c}_{i}
$$

where $i \in\{0,1, \cdots, b-1\}, \hat{\mathbf{c}}_{\mathbf{i}}$ is a constant vector at each iteration, and $\mathbf{c}_{\mathbf{i}}$ is a constant vector at all iterations of the corresponding size as defined in (7). As discussed in Sect. 4, the vector representation of the matrix $D$ is stored in a separated file, which is read $b$ times at each node, the same as a partition of the link file.

Computing $\mathbf{v}_{i}^{\prime}$ at the $i$ th node is carried out in the same fashion as in the serial implementation. It needs to read $L_{i}, \mathbf{v}$ and to write $\mathbf{v}_{\mathbf{i}}^{\prime}$ to update the source vector. The $\mathrm{I} / \mathrm{O}$ cost is $B\left(L_{i}\right)+B(\mathbf{v})+B\left(\mathbf{v}_{i}^{\prime}\right)$. One advantage of the distributed algorithm can be seen in normalizing the destination vector. A serial implementation needs to read the whole destination vector. In the distributed case, each partition of the destination vector is held in memory at its corresponding node and the normalization can be done concurrently. Therefore, the I/O cost at each node is

$$
C_{I / O}=B\left(L_{i}\right)+\left(1+\frac{1}{b}\right) B(\mathbf{v}) \approx B(L) / b+B(\mathbf{v}) .
$$

The in-memory computational cost does not have any parallel overhead and is therefore

$$
C_{\text {in-memory }} \approx C_{2} / b
$$

The communication cost can be a bottleneck. To start a new iteration, every node in the cluster needs to collect one block of the updated source vector from every other node, which is $4 N / b$ bytes. The total communication cost is

$$
C_{\text {comm }}^{\prime}=\sum_{i=0}^{b-1}\left(\sum_{j=0, j \neq i}^{b-1} 4 N / b\right)=4(b-1) N .
$$

By making every pair of all nodes communicate concurrently, the communication cost is reduced to $\mathcal{O}(N)$ since

$$
C_{\text {comm }}=C_{\text {comm }}^{\prime} \times \frac{2}{b} \approx 8 N .
$$

The cost models in (13), (14) and (16) show we could achieve a near linear scale-up and a near linear speed-up provided that the data size, $r N$, is large compared to $N$ since the communication cost is independent of $r$.

\section{Experimental Evaluation}

\subsection{Experimental Setup}

Experiments for the algorithm handling dangling nodes were conducted on Linux platform on a single dedicated machine with a $2.00 \mathrm{GHz} \operatorname{Intel}(\mathrm{R}) \operatorname{Celeron}(\mathrm{R})$ 
Table 1. Data sets and the cost comparison

\begin{tabular}{|c|c|c|c|c|c|c|c|}
\hline Name & Pages & $\mathrm{K} / \mathrm{N}$ & $\mathrm{r}$ & Links & $C_{1}$ & $C_{2}$ & $C_{1} / C_{2}$ \\
\hline California & $9.7 \mathrm{~K}$ & $48.00 \%$ & 1.67 & $16 \mathrm{~K}$ & $26.3 \mathrm{~s}$ & $0.77 \mathrm{~s}$ & $34.2 \mathrm{~s}$ \\
Stanford & $281 \mathrm{~K}$ & $0.06 \%$ & 8.45 & $2.4 \mathrm{M}$ & $58.8 \mathrm{~s}$ & $30.7 \mathrm{~s}$ & $1.9 \mathrm{~s}$ \\
\hline
\end{tabular}

CPU. Experiments for the parallel algorithm were conducted on Windows platform on dedicated machines each with a $2.8 \mathrm{GHz}$ Intel Pentium(R)-4 CPU. The memory size in either case is ample in the sense that there is enough physical memory for the OS. The page size of disk access for all experiments is $512 \mathrm{~KB}$. The implementations of both algorithms were in Java.

Table 1 shows the basic statistics for the two data sets. The first data set, California, is used solely to test the algorithm of handling dangling nodes. It was obtained from http://www.cs.cornell.edu/Courses/cs685/2002fa/. The second data set, stanford, is used for both algorithms. It was obtained from http://www.stanford.edu/ ${ }^{\sim}$ sdkamvar/.

\subsection{Results for Handling Dangling Nodes}

In Tab. 1. $C_{1}$ represents the cost based on the computation model in (3), $C_{2}$ represents the cost based on the computation model in (7), which handles dangling nodes using the proposed algorithm.

The data set california needs only one disk access due to its small size. Its I/O cost is then negligible. The dangling nodes in this data set are almost a half of the total pages. The speedup obtained by the proposed algorithm, which is the ratio of $C_{2}$ and $C_{1}$, is 34.2. This verifies the two cost models in (9) and (10). The data set stanford is about $11.2 M$, which results in about 22 disk accesses. Even though the dangling nodes in the data set are only about $0.06 \%$, the speedup obtained by the proposed algorithm is about 1.9 .

\subsection{Results for the Parallelization of the Algorithm}

The parallel implementation uses the data set stanford only. The experiments were conducted on a cluster of different number of nodes. The experimental results in Tab. 2 show we have achieved a near linear speed-up. One reason for

Table 2. Parallel running times and the corresponding speed-up's

\begin{tabular}{|c|c|c|c|c|}
\hline Number of Nodes & 1 & 2 & 4 & 8 \\
\hline Elapsed Time & $47.1 \mathrm{~s}$ & $23.7 \mathrm{~s}$ & $12.1 \mathrm{~s}$ & $6.2 \mathrm{~s}$ \\
\hline Speed-up & N/A & 2.0 & 3.9 & 7.6 \\
\hline
\end{tabular}


the nice results is that the average out-degree of the experimental data set is 8.45, which makes the data size much larger than the ranking vector size. Therefore, the $\mathrm{I} / \mathrm{O}$ cost and the in-memory computational cost weigh significantly more than the communication cost.

\section{Conclusions and Future Work}

In this paper, we have derived an efficient algorithm and its parallelization for computing PageRank with dangling nodes fully preserved. Both the analysis and the experimental results demonstrate that our algorithm has little overhead associated with inclusion of dangling nodes. There are two areas for future work: conducting experiments on the larger data sets and at a larger parallel cluster; and exploring more fully dangling nodes' impact and their treatment to PagaRank computation.

\section{References}

1. Brin, S., Page, L., Motwami, R., Winograd, T.: The pagerank citation ranking: bringing order to the web. Technical report, Computer Science Department, Stanford University (1999)

2. Langville, A.N., Meyer, C.D.: Deeper inside pagerank. Internet Math 1 (2004) $335-380$

3. Haveliwala, T.H., Kamvar, S.D.: The second eigenvalue of the google matrix. Technical report, Computer Science Department, Stanford University (2003)

4. Elden, L.: A note on the eigenvalues of the google matrix. Report LiTH-MAT-R04-01 (2003)

5. Kamvar, S., Haveliwala, T., Manning, C., Golub, G.: Extrapolation methods for accelerating pagerank computations. Twelfth International World Wide Web Conference (2003)

6. Randall, K., Stata, R., Wickremesinghe, R., Wiener, J.: The link database: Fast access to graphs of the web. In: the IEEE Data Compression Conference. (March 2002)

7. Adler, M., Mitzenmacher, M.: Towards compressing web graphs. In: the IEEE Data Compression Conference. (March 2001)

8. Raghavan, S., Garcia-Molina, H.: Representing web graphs. In: the 19th IEEE Conference on Data Engineering, Bangalore, India (March 2003)

9. Haveliwala, T.H.: Efficient computation of pagerank. Technical report, Computer Science Department, Stanford University (Oct. 1999)

10. Chen, Y., Gan, Q., Suel, T.: I/o-efficient techniques for computing pagerank. In: Proc. of the 11th International Conference on Information and Knowledge Management. (2002)

11. Kamvar, S., Haveliwala, T., Golub, G.: Adaptive methods for the computation of pagerank. Technical report, Stanford University (2003)

12. Lee, C., Golub, G., Zenios, S.: A fast two-stage algorithm for computing pagerank and its extension. Technical report, Stanford University (2003) 